\title{
Rise in Alcohol Related Disorders during COVID 19 Pandemic - An Observation
}

\author{
Rooppreet Gill ${ }^{1}$, Rohit Chordiya ${ }^{*}$, Jitendra D Lakhani ${ }^{2}$
}

${ }^{1}$ Junior Resident, Department of Medicine, SBKSMI\&RC, Sumandeep Vidyapeeth Deemed to be University, Vadodara, Gujrat, India

${ }^{2}$ Professor, Department of Medicine, SBKSMI\&RC, Sumandeep Vidyapeeth Deemed to be University, Vadodara, Gujrat, India

DOI: $10.36347 /$ sasjm.2020.v06i08.002

| Received: 03.08.2020 | Accepted: 12.08.2020 | Published: 19.08.2020

*Corresponding author: Rohit Chordiya

Abstract

As the world is dealing with the rising number of cases of COVID 19 infection and deaths related to it, and number of other psychosocial issues faced among the general population, we have observed a very peculiar and important problem related to increase in substance abuse particularly alcohol and related disorders during this time of crisis. Initially when the disease set its footsteps in India in january,2020, and eventually cases started rising across the other parts of the country, the government took appropriate initiative to combat and halt the spread of the infection across the nation by "complete lockdown" for weeks. Indeed it was an applaudable step but had a negative impact on economic and psychosocial aspects. An unexpected outcome of lockdown was seen among persons addicted to substances like alcohol due to non-availability leading to Alcohol withdrawal syndromes, black marketing of alcohol and increased frustration for no access to alcohol. But later when the process of "unlock" started, there were rising cases of patients admitting into hospitals after binge alcohol drinking for days, presenting as Acute alcoholic hepatitis or Acute Pancreatitis or Acute alcohol intoxication. This article is based on the observation made in our hospital regarding increase in alcohol related diseases attributed to binge drinking related to post-lockdown stress.

Keywords: Stress, covid 19, binge drinking, alcohol, india, mental health.

Copyright @ 2020: This is an open-access article distributed under the terms of the Creative Commons Attribution license which permits unrestricted use, distribution, and reproduction in any medium for non-commercial use (NonCommercial, or CC-BY-NC) provided the original author and source are credited.

\section{INTRODUCTION}

The rapid serious spread of pneumonia due to unknown cause in Wuhan, china, in December 2019 later recognized as coronavirus infection came into global news with fear of spread across the world. It was renamed COVID 19 infection on $11^{\text {th }}$ February 2020 by WHO, and was declared a pandemic within a month, on $11^{\text {th }}$ March, 2020.

The disease first landed in southwestern state of Kerala in India by end of January 2020 from Wuhan [1] and within no less time, it created havoc among the people of the country. The number coronavirus positive cases started rising in India gradually, and the central government decided to take important steps in controlling the spread among which the crucial one was the lockdown after analyzing the situations in other countries like Italy, spain, UK etc. It was the largest lockdown seen, as whole nation came to halt with people at homes, workplaces shut and only essential services opened. The one complex outcome which has medical, ethical, economic and social aspects is related to alcohol consumption. The Lockdown affected the mental health of people as it was a setback for businessmen which lead to increase in stress due to economic loss, the sense of anxiety among normal people related to coronavirus spread, migrant workers being unemployed, rising domestic violence etc. As the process of "Unlock" was deployed, people gained easy access to alcohol, and those who were on "forced abstinence" during lockdown, had relapses leading to binge drinking and dependency.

The other important factor that supposedly leads to binge drinking is the mental stress. There have been various studies done in the past, correlating stressors and binge drinking. It has been found that there is greater alcohol consumption on days when stressful events occurred [2]. Also binge drinking is found to be more likely on days of elevated negative effects suggesting that binge drinking may be the way to cope with elevated distress for the vulnerable people [3].

This paper highlights the effect of stress related to pandemic and increased binge drinking leading to rising trend of alcohol related diseases and 
increased hospital admissions. We have observed this trend in our hospital in previous one month. Based on our observation, we have discussed this important finding further using four clinical cases.

\section{CASE REPORTS}

1) A 31 years old male, chronic alcoholic, farmer by occupation, presented with complaints of abdominal pain from 20 days associated with nausea, vomiting and loss of appetite. He had recent history of binge alcohol drinking for 1 week before developing symptoms, due to financial strains in the family. He had country liquor daily approximately 1 litres per day, at any time of the day. On examination, he had icterus, tachycardia, tachypnea, decreased air entry bilateral lower lung fields on chest auscultation and tenderness in left hypochondrium and epigastric region on palpation. His reports were suggestive of macrocytic anemia, leukocytosis, uremia, hyperbilirubinemia, 3 folds increased serum amylase and lipase and raised ESR and CRP. His chest xray revealed pleural effusion bilateral with basal atelectasis. His abdominal sonography suggested of pancreatitis. CT (Computed Tomography) of abdomen was done which suggested acute pancreatitis with peripancreatic collection. Patient was diagnosed with Severe Alcohol induced Acute Pancreatitis with Multiple organ failure - AKI and type 1 respiratory failure. Patient was managed in ICU and treated with intravenous antibiotics, fluids, hemodialysis and oxygen support. He recovered and was discharged.

2) 38 years/Male, chronic alcoholic, unemployed, was admitted in MICU with complaints of altered sensorium, decreased urinary output and abdominal pain from 2 days. The family gave detailed history of patient's habits and personal life. It was found that patient has been drinking alcohol daily in excessive amounts continuously from last 10-15 days, and has been having labile mood. Based on the clinical examination and laboratory findings, patient was diagnosed with acute alcohol intoxication with Decompensated alcoholic liver disease complicated with portal hypertension, coagulopathy, Acute kidney injury, sepsis and Grade II hepatic encephalopathy. Patient was managed aggressively and was shifted toward after stabilization.

3) A 38 years/Male, Chronic alcohol and tobacco use, a fishmonger by profession, presented with complaints of severe abdominal pain in epigastric region radiating to back, with vomiting and reduced appetite from 4 days. He gave history of stress induced binge alcohol intake for continuous 10-12 days, country liquor, with more than $500 \mathrm{ml}$ in 1-2 hours morning and evening, he also informed us about alcohol abstinence from last 4 years. Patient was relatively stable vitally and was admitted in General medicine ward, and based on his clinical evaluation and investigations, he was diagnosed with Mild Alcohol Induced Acute Pancreatitis without organ failure and local complications. Patient was given supportive treatment. He was counselled and psychiatry consultation was done, and found to have excessive disturbing thoughts about his close relatives who have died recently due to COVID 19 infection and worry about his children.

4) 46 years/Male labourer, chronic alcoholic and smoker, presented with complaints of abdominal distention and pain in epigastrium from 1 month. He gave history of daily intake of country liquor of approximately $500-750 \mathrm{ml}$ and consumption has increased in last 2 months. The cause for this heavy alcohol use was stress from family conflicts related to money lost during lockdown crisis. On examination, he had pallor, signs of liver failure and gross ascites. His Blood reports and abdominal sonography confirmed the diagnosis of Acute decompensated liver cirrhosis with portal hypertension. He was managed in MICU.

\section{ANALYSIS}

The observation made by studying these four cases is clearly suggestive of role of stressors and increased alcohol intake. As we observed that all were male patients with age ranging from 30-50 years. We found stressors related to this pandemic in all the cases. Indeed, all the patients already were having alcohol use disorder but the consumption has increased during the post-lockdown time. As we observed that one patient who has been successfully having alcohol abstinence from last four years, had relapsed due to stress, anxiety and fear related to COVID 19 infection.

All the patients presented after increased alcohol consumption either binge drinking or heavy alcohol use. All of these patients confirmed 'forced abstinence" during the lockdown period.

The psychosocial assessment done in these patients revealed the clear relation between increased substance abuse with increasing stress related to various aspects how this COVID 19 pandemic has affected the lives of people.

\section{DISCUSSION}

Excessive alcohol use is a serious problem and especially during these times of global crisis, it has increased. Binge drinking, as defined by National Institute of Alcohol Abuse and Alcoholism (NIAAA) as a pattern of drinking alcohol that brings blood alcohol concentration (BAC) to 0.08 percent - or 0.08 grams of alcohol per dl - or higher. For a typical adult, this pattern corresponds to consuming 5 or more drinks 
(male), or 4 or more drinks (female), in about 2 hours. And heavy alcohol use is defined as more than 4 drinks on any day for men or more than 3 drinks for women [4].

There are various studies which correlate the acute events, chronic stresses and substance abuse suggesting that the negative affect mediates the relationship between stressful events and alcohol use [5].

COVID 19 pandemic has affected the various aspects of lives, adding to the mental stress. WHO and MOHFW India have been actively working on creating awareness regarding the coping techniques among people suffering from mental distress during the pandemic- "Minding our minds during the COVID-19 [6]".

As we observed in our cases, that alcohol related diseases are rising and the correlation between stress and excessive alcohol use is quite evident. This has increased the burden on healthcare system which is already struggling with rising number of COVID -19 positive cases daily. The alcohol abuse and excessive consumption related disease like Severe acute pancreatitis is itself associated with increased morbidity and mortality, requiring management in ICU settings. And the rising number of cases presenting with such diseases is affecting the healthcare system in negative manner, as we are already facing shortages of healthcare professionals and occupancy.

COVID 19 has already disrupted the economy. Added to it, the burden caused by binge drinking will lead to a greater number of patients, in an already struggling economy. This in turn will impact the economy further. As we have seen in this study, the most affected are the middle-aged adults, who belong to working class. This study merely highlighted or touched upon the surface of an iceberg. Because of the lack of infrastructure or because of the financial conditions, a lot many of these patients do not present themselves to the hospitals. These people not going about their routine activities and working will have a negative impact on the economy.

These conditions which develop as sequelae of binge drinking are critical conditions. Patients will need urgent care and treatment. If these conditions are not treated adequately and early, will lead to mortality. Mindfulness can help in patients having Corona panic [7]. Long term effects, which are not seen immediately, can be a burden to self and to the society. Conditions such as liver cirrhosis, alcohol dependency, chronic pancreatitis etc. are debilitating conditions.

\section{CONCLUSION}

As we have seen how stress related to the various aspects of COVID 19 infection as affected the lives of people especially alcohol dependant patients leading to binge drinking and heavy alcohol use. This has increased the burden on healthcare system as the number of cases of COVID 19 is rising, so are the stress and alcohol consumption and eventually alcohol related disorders. Our cases as described above prove the fact and correlation between the rising stress, alcohol consumption and its consequences on the physical and mental health.

\section{REFERENCES}

1. Rawat M. Coronavirus in India: Tracking country's first 50 COVID-19 cases; what numbers tell. India Today. March. 2020 Mar; 12.

2. Armeli S, Carney MA, Tennen H, Affleck G, O'Neil TP. Stress and alcohol use: A daily process examination of the stressor-vulnerability model. Journal of personality and social psychology. 2000 May;78(5):979.

3. Swendsen JD, Tennen H, Carney MA, Affleck G, Willard A, Hromi A. Mood and alcohol consumption: an experience sampling test of the self-medication hypothesis. Journal of abnormal psychology. 2000 May;109(2):198.

4. Cooper ML, Russell M, Skinner JB, Frone MR, Mudar P. Stress and alcohol use: moderating effects of gender, coping, and alcohol expectancies. Journal of abnormal psychology. 1992 Feb;101(1):139.

5. Armeli S, Carney MA, Tennen H, Affleck G, O'Neil TP. Stress and alcohol use: A daily process examination of the stressor-vulnerability model. Journal of personality and social psychology. 2000 May;78(5):979.

6. https://www.mohfw.gov.in/pdf/Mindingourmindsd uringCoronaeditedat.pdf./9thAug 2020.

7. Janki B Patel, J D Lakhani. How mindfulness can help you to sail through corona panic. Tathapi. 2020; 19(28). 